\title{
Low Voltage Nanotip Interferometry
}

A. Thesen ${ }^{*}$, B.G. Frost ${ }^{*}$, and D.C. Joy ${ }^{*}$

*EM Facility, University of Tennessee, Knoxville, TN 37996 and High Temperatures Materials Laboratory, Oak Ridge National Laboratory, Bethel Valley Road 1, Oak Ridge, TN 37831

The point projection microscope ( $\mathrm{ppm})$ is unique in that it contains no optical elements. The basic ppm, which was first described by Morton and Ramberg in 1939 [1], consists of just three components shown schematically in figure 1: A source of electrons, thespecimen and the detector. The cone of illumination strikes the object to produce a shadow image, which is magnified by the ratio of the distance between the source and the detector (Y) to that between the source and the specimen $(\mathrm{X})$. Because this instrument contains no lenses it has no distortions and is not limited by electron-optical aberrations. In fact, the ultimate resolution is set only by the size of the electron source and the wavelength of the electrons.

Since all properties of the microscope are controlled by the quality of the nanotip field emitters our main task was their development and the characterization of their performance. We now can produce nanotips by etching tungsten wires, which show turn on energies well below 300V. Our tip brightness at $500 \mathrm{~V}$ was measured to be about $10^{7} \mathrm{~A} /(\mathrm{cm} 2 \mathrm{str})$, which is enough to allow TV rate imaging for a 512 by 512 pixel image. Stable tip performance requires UHV conditions because of the parallel image acquisition, however, the tip stability is not as critical as on scanning microscopes and even at $10^{-8}$ Torr a satisfactory emission can be obtained.

Since our field emission tips exhibit a high degree of coherency the resulting image is an inline Fresnel hologram containing true 3D information of the imaged object. The resulting prospects of this for tip interferometry have only been discussed recently [2]. The main problem is the difficulty of reconstructing inline holograms [3]. Figure 2 shows an image of a holey carbon film in transmission recorded at a beam energy of $350 \mathrm{eV}$ and $30 \mathrm{~ms}$ exposure. Several kinds of diffraction effects are evident: The holes are outlined by several orders of Fresnel fringes. In addition there are fringes along and across the bridges between the holes of variable spacing and contrat, some of which show a significantly higher brightness than the surrounding images features. These patterns can be explained by assuming the bridge charges up under the beam and acts like a Moellenstedt biprism overlapping the two parts of the wavefront. Particularly interesting are the points at which different types of fringes connect.

References

[1] G.A. Morton and E.G. Ramberg, Phys. Rev. 56 (1939), p.705

[2] V. Georges et al., Ultramicroscopy 90 (2001), p.33-38

[3] J.C. Spence, et al. in Electron Holography, A. Tonomura et al. (Editors), 1995 Elsevier Sciences, p. 267-276

[4] This research was supported by SRC under contract number LJ413.003 and was carried out as part of the user program sponsored by the U.S. Department of Energy under contract DE AC05-00OR22725 with the Oak Ridge National Laboratory, managed by UT-Battelle, LLC. 


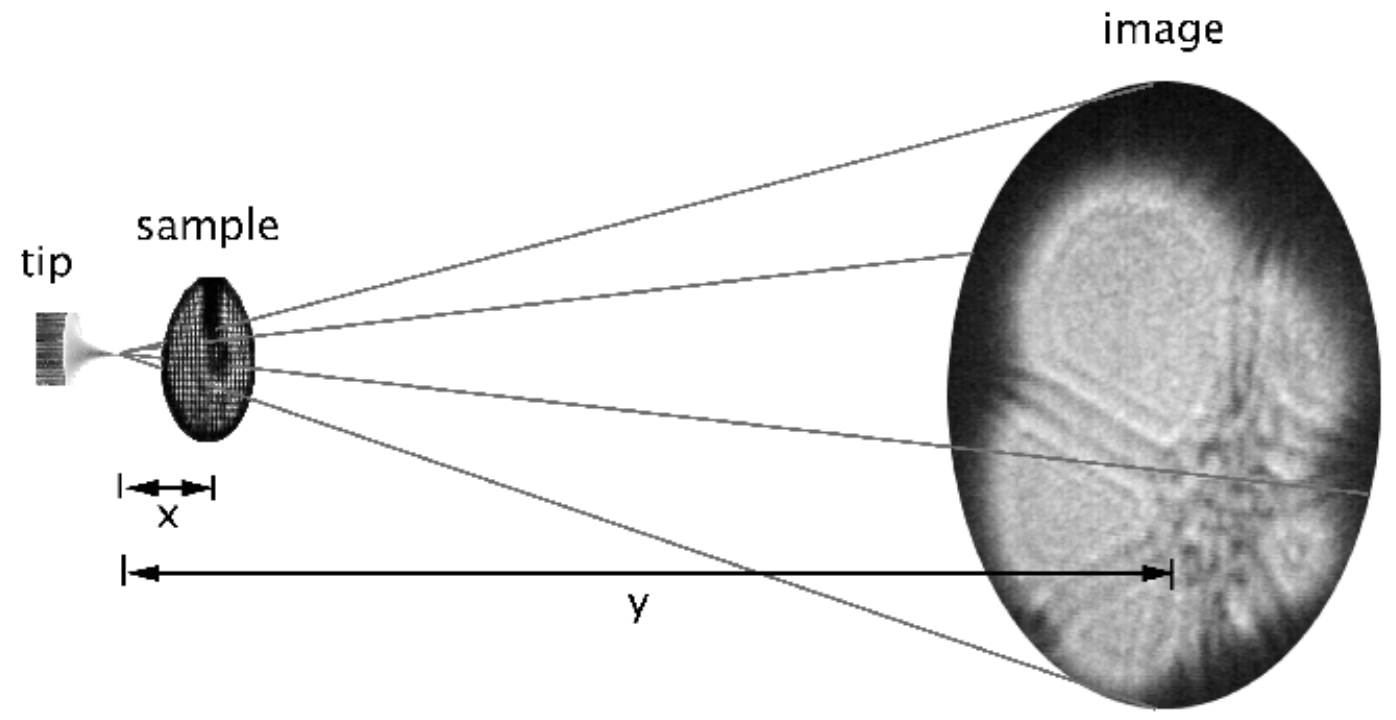

FIG. 1. Basic sketch of a point projection microscope

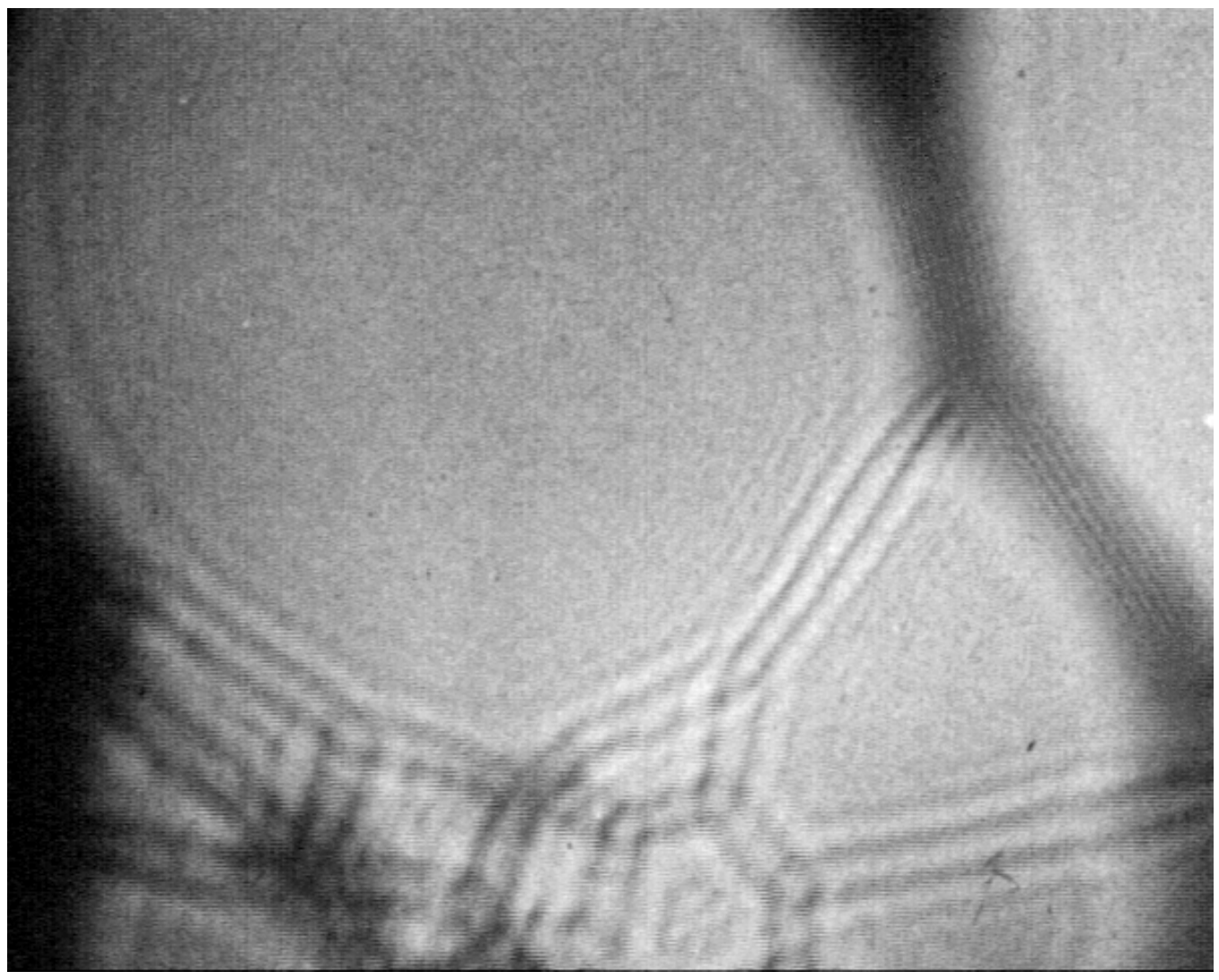

FIG. 2. Point projection image of a holy carbon foil at $350 \mathrm{eV}$ showing complex interference patterns 